\title{
CAUCHY PROBLEM FOR A LINEAR HYPERBOLIC EQUATION OF THE SECOND ORDER
}

\author{
V.I.KORZYUK and E.S. CHEB \\ Belarussian State University \\ Skoriny 4, 220050, Minsk, Belarus \\ E-mail: korzyuk@suas . bas-net.by; cheb@bsu.by \\ Received November 27, 2005; revised April 26, 2006; published online September 15, 2006
}

\begin{abstract}
The definition of hyperbolic equation by a prescribed vector field is introduced for linear differential equation of the second order. The Cauchy problem with prescribed boundary conditions is considered for such equations. The theorems of existence and uniqueness of a strong solution to the given problem are proved by the method of energy inequalities and mollifiers with variable step.
\end{abstract}

Key words: hyperbolic equation, Cauchy problem, strong solution, energy inequality, mollifiers

\section{Introduction}

Well posedness of boundary value problems are of interest in the theory of partial differential equations. Investigation of correctness is connected to the proof of existence and uniqueness of the solution to the problem. One of the generally used methods is a functional analysis method which is based on the investigation of reversibility of the operator associated to the initial problem $[3,8]$. The proof of the uniqueness of a strong solution [2] assumes acquisition of an assessment for the required solution by the use of the problem operator value. This is so called energy estimate. The proof of the existence is based on the investigation of a conjugate problem by applying to it mollifiers with a variable step [1].

The bibliography about Cauchy problem for a linear hyperbolic equations is very extensive (see, for a example, $[4,6]$ and bibliography in [6]). In this article hyperbolic equations by a prescribed vector field are considered and the new method of energy inequalities and mollifiers with variable step is proposed for investigation of correctness of boundary problems in the theory of partial differential equations. 


\section{Definition of the Hyperbolic Equation}

We consider the linear partial differential equation

$$
\mathcal{L}(x, \mathbf{D}) u \equiv \sum_{|\alpha| \leqslant 2} a_{\alpha}(x) \mathbf{D}^{\alpha} u=f(x),
$$

where $u, a_{\alpha}, f: \mathbb{R}^{n} \supset Q \ni x \rightarrow u(x), a_{\alpha}(x), f(x) \in \mathbb{R}$ are functions of independent $n$ variables $x=\left(x_{1}, \ldots, x_{n}\right)$. The functions $u, a_{\alpha}, f$ are defined in the domain $Q \subset \mathbb{R}^{n}$ of $n$ dimensional Euclidean space $\mathbb{R}^{n}$. Here

$$
\mathbf{D}^{\alpha}=\mathrm{D}_{1}^{\alpha_{1}} \ldots \mathrm{D}_{n}^{\alpha_{n}}=\frac{\partial^{|\alpha|}}{\partial x_{1}^{\alpha_{1}} \ldots \partial x_{n}^{\alpha_{n}}}, \quad \alpha=\left(\alpha_{1}, \ldots, \alpha_{n}\right)
$$

is a multi-index, $|\alpha|=\alpha_{1}+\ldots+\alpha_{n}$, where $\alpha_{j}(j=1, \ldots, n)$ are nonnegative integers.

Vector field $\mathcal{N}$ is of the class $C^{1}$, if the functions $\eta_{k}(x)(k=1, \ldots, n)$ belong to the class $C^{1}\left(\mathbb{R}^{n}\right)$. Suppose that vector field $\mathcal{N}$ of the class $C^{1}$ is defined in $\mathbb{R}^{n}$ and consists of elements-unit vectors

$$
\boldsymbol{\eta}(x)=\left(\eta_{1}(x), \ldots, \eta_{n}(x)\right), \quad|\boldsymbol{\eta}|^{2}=\eta_{1}^{2}+\ldots+\eta_{n}^{2}=1 .
$$

Definition 1. Equation (2.1) will be referred to as hyperbolic in point $x$ with respect to the direction $\boldsymbol{\eta}(x)$ if

(i) polynomial $\mathcal{L}_{0}(x, \boldsymbol{\eta}(x))=\sum_{|\alpha|=2} a_{\alpha}(x) \eta_{1}^{\alpha_{1}}(x) \ldots \eta_{n}^{\alpha_{n}}(x) \neq 0$; here for definiteness we assume $\mathcal{L}_{0}(x, \boldsymbol{\eta}) \geqslant \delta, \delta$ is some positive integer;

(ii) polynomial $\mathcal{L}_{0}(x, \tau \boldsymbol{\eta}(x)+\boldsymbol{\xi}(x))$ with respect to $\tau \in \mathbb{R}^{1}$ has two real different roots for any

$$
\boldsymbol{\xi}(x)=\left(\xi_{1}(x), \ldots, \xi_{n}(x)\right),|\boldsymbol{\xi}(x)|=1,(\boldsymbol{\eta}(x), \boldsymbol{\xi}(x))=\sum_{k=1}^{n} \eta_{k}(x) \xi_{k}(x)=0 .
$$

Equation (2.1) is hyperbolic in closure $\bar{Q} \subset \mathbb{R}^{n}$ of domain $Q$, if it is hyperbolic in each point $x \in \bar{Q}$ with respect to the direction $\boldsymbol{\eta}(x)$ from the vector field $\mathcal{N}$. For convenience we will write the expression $\mathcal{L}(x, \mathbf{D}) u$ in the following form

$$
\begin{aligned}
& \mathcal{L}(x, \mathbf{D}) u=\sum_{i, j=1}^{n}\left(a_{i j}(x) u_{x_{i}}\right)_{x_{j}}+\sum_{i=1}^{n} a_{i}(x) u_{x_{i}}+a_{0}(x) u, \\
& u_{x_{i}}=\frac{\partial u}{\partial x_{i}}, \quad u_{x_{i} x_{j}}=\frac{\partial^{2} u}{\partial x_{i} \partial x_{j}}, \quad a_{i j}=a_{j i}(i, j=1, \ldots, n), \\
& \mathcal{L}_{0}(x, \mathbf{D})=\sum_{i, j=1}^{n} a_{i j}(x) \frac{\partial^{2}}{\partial x_{i} \partial x_{j}} .
\end{aligned}
$$

We will designate the cone $K(x)$ as a set of vectors $\zeta(x)$, for which $\mathcal{L}_{0}(x, \zeta(x)) \geqslant 0$. This set can be described by relations 


$$
\begin{aligned}
& \boldsymbol{\zeta}(x)=\mu(\tau \boldsymbol{\eta}(x)+\boldsymbol{\xi}(x)), \quad \boldsymbol{\zeta}(x)=\mu \boldsymbol{\eta}(x), \quad \mu \in[0, \infty), \tau \in \mathbb{R}^{1} \\
& \tau \mathcal{L}_{0}(x, \boldsymbol{\eta}) \geqslant-\mathcal{L}_{0}(x ; \boldsymbol{\eta}, \boldsymbol{\xi})+G^{1 / 2}(x, \boldsymbol{\eta}, \boldsymbol{\xi}), \\
& \mathcal{L}_{0}(x ; \boldsymbol{\eta}, \boldsymbol{\xi})=\sum_{i, j=1}^{n} a_{i j}(x) \eta_{i}(x) \xi_{j}(x), \\
& G(x ; \boldsymbol{\eta}, \boldsymbol{\xi})=\mathcal{L}_{0}^{2}(x ; \boldsymbol{\eta}, \boldsymbol{\xi})-\mathcal{L}_{0}(x, \boldsymbol{\eta}) \mathcal{L}_{0}(x, \boldsymbol{\xi}),
\end{aligned}
$$

and vectors $\boldsymbol{\eta}(x)$ and $\boldsymbol{\xi}(x)$ are from the definition 1. Let us note that $\tau \boldsymbol{\eta}(x) \in$ $K(x)$ for all $\tau \in[0, \infty)$.

Proposition 1. Cone $K(x)$ is a convex set.

Proof Let $\boldsymbol{\eta}(x)=(\tau \boldsymbol{\eta}+\boldsymbol{\xi})(x)$ and $\widetilde{\boldsymbol{\eta}}(x)=(\widetilde{\tau} \boldsymbol{\eta}+\widetilde{\boldsymbol{\xi}})(x)$ be an arbitrary point of the cone $K(x)$. Hence,

$$
\begin{aligned}
& \tau \mathcal{L}_{0}(x, \boldsymbol{\eta}) \geqslant-\mathcal{L}_{0}(x ; \boldsymbol{\eta}, \boldsymbol{\xi})+G^{1 / 2}(x ; \boldsymbol{\eta}, \boldsymbol{\xi}), \\
& \widetilde{\tau} \mathcal{L}_{0}(x, \boldsymbol{\eta}) \geqslant-\mathcal{L}_{0}(x ; \boldsymbol{\eta}, \widetilde{\boldsymbol{\xi}})+G^{1 / 2}(x ; \boldsymbol{\eta}, \widetilde{\boldsymbol{\xi}}),
\end{aligned}
$$

where $(\boldsymbol{\eta}, \boldsymbol{\xi})=0$ and $(\boldsymbol{\eta}, \widetilde{\boldsymbol{\xi}})=0 ; \boldsymbol{\eta}, \widetilde{\boldsymbol{\xi}} \in \mathbb{R}^{n}$. Note that the set $M=\{\boldsymbol{\xi}(x) \in$ $\left.\mathbb{R}^{n} \mid(\boldsymbol{\eta}, \boldsymbol{\xi})=0\right\}$ is a subspace of space $\mathbb{R}^{n}$. It follows from the definition 1 that the expression $G^{1 / 2}(x ; \boldsymbol{\eta}, \boldsymbol{\xi})$ is the element norm, therefore CauchyBunyakovsky inequality is fulfilled

$$
|G(x ; \boldsymbol{\eta}, \boldsymbol{\xi}, \widetilde{\boldsymbol{\xi}})| \leqslant G^{1 / 2}(x ; \boldsymbol{\eta}, \boldsymbol{\xi}) G^{1 / 2}(x ; \boldsymbol{\eta}, \widetilde{\boldsymbol{\xi}})
$$

for a scalar product

$$
G(x ; \boldsymbol{\eta}, \boldsymbol{\xi}, \widetilde{\boldsymbol{\xi}})=\mathcal{L}_{0}(x ; \boldsymbol{\eta}, \boldsymbol{\xi}) \mathcal{L}_{0}(x ; \boldsymbol{\eta}, \widetilde{\boldsymbol{\xi}})-\mathcal{L}_{0}(x ; \boldsymbol{\eta}) \mathcal{L}_{0}(x ; \boldsymbol{\xi}, \widetilde{\boldsymbol{\xi}})
$$

with respect to elements $\boldsymbol{\xi}, \widetilde{\boldsymbol{\xi}} \in M$. The fact that the equation (2.5) prescribes a scalar product follows from the condition of hyperbolicity of the equation (2.1). Hence, according to the definition of cone $K(x)$, it is sufficient to prove the inequality

$$
\begin{aligned}
(\lambda \tau(x)+(1-\lambda) \widetilde{\tau}(x)) \mathcal{L}_{0}(x ; \boldsymbol{\eta}) \geqslant-\mathcal{L}_{0}(x & ; \boldsymbol{\eta}, \lambda \boldsymbol{\xi}+(1-\lambda) \widetilde{\boldsymbol{\xi}}) \\
& +G^{1 / 2}(x ; \boldsymbol{\eta}, \lambda \boldsymbol{\xi}+(1-\lambda) \widetilde{\boldsymbol{\xi}})
\end{aligned}
$$

for any $\lambda \in[0,1]$. It is easy to check that

$$
\begin{aligned}
\mathcal{L}_{0}(x ; \boldsymbol{\eta}, \lambda \boldsymbol{\xi}+(1-\lambda) \widetilde{\boldsymbol{\xi}})=\lambda \mathcal{L}_{0}(x ; \boldsymbol{\eta}, \boldsymbol{\xi})+(1-\lambda) \mathcal{L}_{0}(x ; \boldsymbol{\eta}, \widetilde{\boldsymbol{\xi}}) \\
G(x ; \boldsymbol{\eta}, \lambda \boldsymbol{\xi}+(1-\lambda) \widetilde{\boldsymbol{\xi}})=\lambda^{2} G(x ; \boldsymbol{\eta}, \boldsymbol{\xi})+2 \lambda(1-\lambda) G(x ; \boldsymbol{\eta}, \boldsymbol{\xi}, \widetilde{\boldsymbol{\xi}}) \\
+(1-\lambda)^{2} G(x ; \boldsymbol{\eta}, \widetilde{\boldsymbol{\xi}}) .
\end{aligned}
$$

From here and from the inequalities (2.2), (2.3) using (2.4) we prove inequality (2.6).

Also we consider a cone $K^{\perp}(x)$, which is dual with respect to the cone $K(x)$. Vectors $\gamma(x)=\left(\gamma_{1}(x), \ldots, \gamma_{n}(x)\right) \in K^{\perp}(x)$ are defined by a scalar product $(\gamma(x), \boldsymbol{\zeta}(x))=\sum_{k=1}^{n} \gamma_{k}(x) \zeta_{k}(x) \geqslant 0$ for any vector $\boldsymbol{\zeta}(x) \in K(x)$. 
Proposition 2. Cone $K^{\perp}(x)$ is a convex set.

Proof The proof follows from the proposition 1 and the definition of the convexity.

\section{Statement of Cauchy Problem}

Suppose that boundary $\partial Q$ of the domain $Q$ is piecewise smooth. By using characteristic polynomial $\mathcal{L}_{0}(x, \boldsymbol{\xi}(x))$, vectors $\boldsymbol{\eta}(x) \in \mathcal{N}$, and external normals $\boldsymbol{\nu}(x)(x \in \partial Q)$ we shall divide $\partial Q$ into different parts, in which different type of Cauchy conditions will be prescribed. Therefore, in addition to the vector field $\mathcal{N}$, we introduce vector field $\Re$ with the help of the cone $K^{\perp}(x)$. By $\Re$ we denote the vector field in $\mathbb{R}^{n}$ of elements $\boldsymbol{r}(x)=\left(r_{1}(x), \ldots, r_{n}(x)\right)$, which are defined by the following conditions:

$\left(\Re_{1}\right)$ for every point $x \in Q$ vector $\boldsymbol{r}(x)$ is the vector of the cone $K^{\perp}(x)$;

$\left(\Re_{2}\right)$ for every point $x \in Q$ and unit vector $\boldsymbol{\xi}(x) \in \overline{K(x)}$ scalar product

$$
r_{\xi}=(\boldsymbol{r}(x), \boldsymbol{\xi}(x))=\sum_{k=1}^{n} r_{k}(x) \xi_{k}(x) \geqslant \delta>0
$$

$\left(\Re_{3}\right)$ field $\Re$ belongs to class $C^{1}$.

Let $\boldsymbol{\nu}(x)$ be a unit vector of the external normal to the domain $\partial Q$ perpendicular to hypersurface $\partial Q$ in point $x \in \partial Q$. We denote by $r_{\nu}$ the scalar product

$$
r_{\nu}=(\boldsymbol{r}(x), \boldsymbol{\nu}(x))=\sum_{k=1}^{n} r_{k}(x) \nu_{k}(x) .
$$

Proposition 3. Vector field $\Re$, defined with respect to the operator $\mathcal{L}(x, \mathbf{D})$, is such that for any $x \in \partial Q$ each vector $\boldsymbol{q}(x)=\left(q_{1}(x), \ldots, q_{n}(x)\right)$, which is perpendicular to $\boldsymbol{r}(x) \in \Re$, satisfies the inequality

$$
\mathcal{L}_{0}(x, \boldsymbol{q}(x)) \leqslant-\delta, \quad|\boldsymbol{q}(x)|=1 .
$$

Proof This proposition follows from the definition of sets $\Re$ and $K^{\perp}(x)$ and conditions $\left(\Re_{1}\right),\left(\Re_{2}\right)$ and $\left(\Re_{3}\right)$.

In the general case let us suppose that the boundary $\partial Q$ consists of the following part:

$$
\partial Q=\left\{x \in \partial Q \mid \mathcal{L}_{0}(x ; \boldsymbol{\nu}(x)) \geqslant \delta, r_{\nu}(x)<0\right\} .
$$

We add to equation (2.1) the following boundary Cauchy conditions:

$$
l_{0} u=\left.u\right|_{\partial Q}=\varphi(x), \quad l_{1} u=\left.\frac{\partial u}{\partial \boldsymbol{p}}\right|_{\partial Q}=\psi(x), \quad x \in \partial Q
$$

where $\partial / \partial \boldsymbol{p} \in \mathcal{P}$ is a derivative along $\boldsymbol{p}$, vector field $\mathcal{P}$ is of the class $C^{1}$ and is not tangent to hypersurface $\partial Q$. 
Problem (2.1), (3.1) can be considered as operator equation

$$
L u=F,
$$

where $L u=\left(\mathcal{L}(x, \mathbf{D}) u, l_{0} u, l_{1} u\right), F=(f(x), \varphi(x), \psi(x))$, the domain of definition of operator $L$ is $\mathcal{D}(L)=C^{2}(\bar{Q})$, where $C^{2}(\bar{Q})$ is a set of twice continuously differentiable functions in $\bar{Q}$.

\section{Strong Solution}

Let us suppose that the problem is written in the form of linear operator equation

$$
L u=F,
$$

where operator $L$ is defined in Banach space $B$ and acts into Hilbert space $H$. By energy inequality the inequality of the form

$$
\|u\|_{B} \leqslant c\|L u\|_{H}
$$

is satisfied for any function $u$ from the domain $\mathcal{D}(L)$, which is dense in $B$, constant $c>0$ is independent on $u ;\|\cdot\|_{B},\|\cdot\|_{H}$ denote norms in spaces $B$ and $H$ respectively.

Suppose that operator $L$ of equation (4.1) allows closure $\bar{L}$. It is well known $[1,11]$ that linear operator $L: B \supset \mathcal{D}(L) \ni u \rightarrow L u \in H$ allows closure $\bar{L}$ if and only if equality $F=0$ follows from $u_{k} \rightarrow 0$ in $B\left(u_{k} \in \mathcal{D}(L)\right)$ and $L u_{k} \rightarrow F$ in $H$.

Definition 2. Solution to the operator equation

$$
\bar{L} u=F, \quad u \in \mathcal{D}(L),
$$

is a strong solution of equation (4.1)

Theorem 1. If the energy inequality (4.2) is valid for operator $L: B \rightarrow H$ and operator $L$ allows closure $\bar{L}$, then the energy inequality

$$
\|u\|_{B} \leqslant c\|\bar{L} u\|_{H}
$$

is valid for any element $u \in \mathcal{D}(\bar{L})$ with the constant $c>0$ from the inequality (4.2).

Statement of the theorem is in fact a corollary of inequality (4.2) and a definition of operator $\bar{L}$. Inequality (4.3) is derived from (4.2) by limit passage for any function $u \in \mathcal{D}(\bar{L})$.

From inequality (4.3) and linearity of the operator $\bar{L}$, uniqueness of strong solution of equation (4.1) follows, if it exists. Inequality (4.2) is a criterion of continuity of inverse operator $L^{-1}$, defined on a set of values $\mathcal{R}(L)$ of operator $L$. Continuous operator $L^{-1}$ can be extended by continuity to the set $\overline{\mathcal{R}(L)}$ (closure $\mathcal{R}(L)$ ). As a result of the extension we obtain continuous operator $\overline{L^{-1}}$ on the set $\overline{\mathcal{R}(L)}$. 


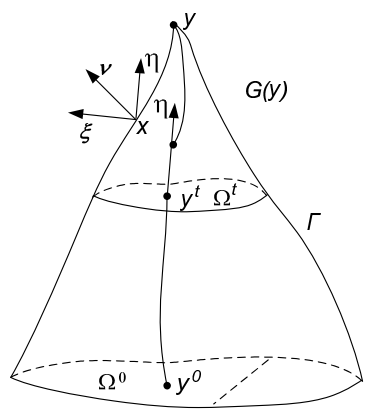

Figure 1. Domain $G(y)$.

Theorem 2. If energy inequality (4.2) holds for the operator $L: B \rightarrow H$, and operator $L$ allows closure $\bar{L}$, then $\overline{\mathcal{R}(L)}=\mathcal{R}(\bar{L})$ and $\overline{L^{-1}}=\bar{L}^{-1}$, where $\bar{L}^{-1}$ is inverse operator with respect to operator $\bar{L}$, defined on a set of values $\mathcal{R}(\bar{L})$ of the operator $\bar{L}$.

Proof Based on its definition $\overline{\mathcal{R}(L)} \supset \mathcal{R}(\bar{L})$. We now prove inverse inclusion, i. e. $\overline{\mathcal{R}(L)} \subset \mathcal{R}(\bar{L})$. Let $F \in \overline{\mathcal{R}(L)}$. There is a sequence $\left\{F_{k}\right\}_{k=1}^{\infty}, F_{k} \in \mathcal{R}(L)$, which converges to $F$ in $H$ when $k \rightarrow \infty$. The sequence $\left\{F_{k}\right\}_{k=1}^{\infty}$ is fundamental and $F_{k}=L u_{k}, u_{k} \in \mathcal{D}(L)$. From inequality (4.2) it follows that the sequence $\left\{u_{k}\right\}_{k=1}^{\infty}$ is fundamental in $B$. Since $B$ is a Banach space, then there exists $u \in B$ and $u_{k} \rightarrow u$ in $B$. This means that according to strong solution definition, $u \in \mathcal{D}(\bar{L})$ and $\bar{L} u=F$, i.e. $F \subseteq \mathcal{R}(\bar{L})$. From here and from the equality $\mathcal{D}\left(\overline{L^{-1}}\right)=\mathcal{D}\left(\bar{L}^{-1}\right)$ it follows that $\overline{L^{-1}}=\bar{L}^{-1}$.

Corollary 1. In order to prove existence of a strong solution to the equation (4.1) with any $F \in H$, it is sufficient to prove the inequality (4.2), existence of closure of operator $L$ and density of a set of values $\mathcal{R}(L)$ in space $H$.

\section{Strong Solution to Cauchy Problem (2.1), (3.1)}

In domain $Q$ we considered arbitrary point $y=\left(y_{1}, \ldots, y_{n}\right)$. To this point, according to definition 1 , corresponds vector $\boldsymbol{\eta}(y)$, where $\mathcal{L}_{0}(y, \boldsymbol{\eta}(y))>0$ and two vectors $\boldsymbol{\zeta}^{ \pm}(y)=\boldsymbol{\tau}_{ \pm} \boldsymbol{\eta}(y)+\boldsymbol{\xi}(y)$, where

$$
\mathcal{L}_{0}\left(y ; \boldsymbol{\zeta}^{ \pm}(y)\right)=0, \quad \boldsymbol{\tau}_{ \pm}=-\mathcal{L}_{0}(y ; \boldsymbol{\eta}, \boldsymbol{\xi}) \pm G^{1 / 2}(y ; \boldsymbol{\eta}, \boldsymbol{\xi}) / \mathcal{L}_{0}(y ; \boldsymbol{\eta}(y))
$$

for any vector $\boldsymbol{\xi}(y)$, orthogonal to vector $\boldsymbol{\eta}(y)$. Designate by $G(y)=G$ the subset of the domain $Q$, which is shown in Fig. 1, and has the form of curvilinear cone with the top in point $y$, base $\Omega^{0}$, and lateral surface $\Gamma$. Let $\bar{G}$ be a closure of the set $G$. Hence, $\Omega^{0}=\bar{G} \cap \partial Q$. Lateral surface $\Gamma$ is characteristic with respect to operator $\mathcal{L}(x, \mathbf{D})$.

It means the following: the normal vector $\boldsymbol{\nu}(x)$ to hypersurface $\Gamma$ at any point $x \in \Gamma$ satisfies the characteristic equation 
Cauchy Problem for a Linear Hyperbolic Eq. of the Second Order 281

$$
\mathcal{L}_{0}(x, \boldsymbol{\nu}(x))=\sum_{|\boldsymbol{\alpha}|=2} a_{(\boldsymbol{\alpha})}(x) \boldsymbol{\nu}_{1}^{\boldsymbol{\alpha}_{1}}(x) \ldots \boldsymbol{\nu}_{n}^{\boldsymbol{\alpha}_{n}}(x)=0 .
$$

For any point $x \in \Gamma$ from $\left(\Re_{2}\right)$ the following condition is satisfied

$$
r_{\nu}=(\boldsymbol{r}(x), \boldsymbol{\nu}(x))=\sum_{k=1}^{n} r_{k}(x) \nu_{k}(x) \geqslant \delta>0 .
$$

The line in Fig. 1, passing through points $y, y^{(t)}$ and $y^{(0)}$, is generated by the field $\mathcal{N}$ in the sense that a tangent to this line in any point coincides with vector $\boldsymbol{\eta} \in \mathcal{N}$.

Now we consider equation (2.1)

$$
\mathcal{L}_{0}(x ; \mathbf{D}) u=f(x), \quad x \in G,
$$

in a set $G(y)=G \subset Q$ with Cauchy condition on $\Omega_{0}$

$$
l_{0} u=\left.u\right|_{\Omega^{0}}=\varphi(x), l_{1} u=\left.\frac{\partial u}{\partial \mathbf{p}}\right|_{\Omega^{0}}=\psi(x), x \in \Omega^{0} .
$$

For problem (5.1)-(5.2) let us consider a space $C^{2}(\bar{G})$ as the domain of definition $\mathcal{D}(L)$ of the operator $L=\left(\mathcal{L}, l_{0}, l_{1}\right)$. We introduce functional spaces $B$ and $H$ for problem (5.1)-(5.2).

By $\Omega(x)$ we denote a section of set $\bar{G}$ such that:

1. $\mathcal{L}_{0}(x, \boldsymbol{\nu}(z))>\delta>0$ for almost all points $x \in \Omega(x)$, where $\boldsymbol{\nu}(z)$ is a unit normal vector to the surface $\Omega(x)$ at point $z \in \Omega(x)$.

2. $\Omega(x)$ is a piecewise smooth hypersurface such that its smooth parts are surfaces of the class $C^{1}$.

3. The family of the sections $\{\Omega(x)\}_{x \in \bar{G}}$ is such that two different sections from the set do not intersect at any point $x \in \bar{G}$, i. e. the points of the same section are on the one side with respect to the other section.

To each section $\Omega(x)$ we assign the parameter $t \in[0,1]$ and denote by $\Omega^{t}$ the section corresponding to the parameter $t$. Suppose that

1. $\bar{G}=\cup_{0 \leqslant t \leqslant 1} \Omega^{t}$.

2. For different $t \neq \tilde{t}(t, \tilde{t} \in[0,1]), \Omega^{t} \cap \Omega^{\tilde{t}}=\varnothing$.

3. Hypersurface $\left\{\Omega^{t}\right\}_{t \in[0,1]}=\{\Omega(x)\}_{x \in \bar{G}}$ and $y \in \Omega^{1}, \Omega^{0} \subset \partial Q$.

By $B$ we denote Banach space, obtained by the closure of the set $\mathcal{D}(L)$ in the norm

$$
\|u\|_{B}=\sup _{0 \leqslant t \leqslant 1} \sum_{|\alpha| \leqslant 1}\|\mathbf{D} u\|_{L_{2}\left(\Omega^{t}\right)},
$$

where $\|\cdot\|_{L_{2}\left(\Omega^{t}\right)}$ is the norm in the space of Lebesgue space square integrable functions defined on the surface $\Omega^{t}$. By $H$ we denote Hilbert space

$$
H=L_{2}(G) \times H^{1}\left(\Omega^{0}\right) \times L_{2}\left(\Omega^{0}\right),
$$

where $H^{1}\left(\Omega^{0}\right)$ is Sobolev space of Lebesgue square integrable in $\Omega^{0}$ functions which possess the square integrable generalized derivatives of the first order. 
Presumption 1. Coefficients $a_{\alpha}(x)$ of equation (2.1) are sufficiently smooth, that is $a_{\alpha}(x) \in C^{2}(\bar{G})$ for $|\alpha|=2$ and $a_{\alpha}(x)$ are measurable and bounded for $|\alpha| \leqslant 1$.

Theorem 3. If presumption 1 holds and $F=(f, \varphi, \psi) \in H$, then there exists the unique strong solution $u$ of problem (5.1)-(5.2) and the estimate

$$
\|u\|_{B} \leqslant c\|F\|_{H}
$$

is true.

Proof To prove this statement it is sufficient to prove the energy inequality

$$
\|u\|_{B} \leqslant c\|L u\|_{H}=c\left(\|\mathcal{L} u\|_{L_{2}(G)}+\left\|l_{0} u\right\|_{H^{1}\left(\Omega^{0}\right)}+\left\|l_{1} u\right\|_{L_{2}\left(\Omega^{0}\right)}\right)
$$

for any function $u \in \mathcal{D}(L)=C^{2}(\bar{G})$, next prove that the operator $L: B \rightarrow H$ admits a closure and to show that the density of a set of values $\mathcal{R}(L) \in H$, where constant $c>0$ in (5.5) does not depend on $u$ and spaces $B$ and $H$ are defined by relations (5.3) and (5.4). The last statement defined by the consequence 1 we will prove later.

Note, if Theorem 3 is proved, the existence and uniqueness of a strong solution of problem (2.1), (3.1) is also proved. In fact, any point $x \in Q$ belongs to some respective cone $G \subset Q$ with the base $\Omega^{0}$ on the boundary $\partial Q$ of the domain $Q$. Then, for any functions $f: Q \ni x \rightarrow f(x) \in \mathbb{R}^{1}, \varphi: \partial Q \ni x \rightarrow$ $\varphi(x) \in \mathbb{R}, \psi: \partial Q \ni x \rightarrow \psi(x) \in \mathbb{R}$, which are narrow on sets $G$ and $\Omega^{0}$ and belong accordingly to $L_{2}(G), H^{1}\left(\Omega^{0}\right)$, and $L_{2}\left(\Omega^{0}\right)$, there exists the unique strong solution $u \in B$ with the norm (5.3).

\section{Energy Inequality for Solutions of Problem (5.1)-(5.2)}

Theorem 4. If presumption 1 is satisfied for the operator $\mathcal{L}$ of equation (5.1), then the energy inequality

$$
\|u\|_{B} \leqslant c\|L u\|_{H}
$$

holds for any functions $u \in \mathcal{D}(L)=C^{2}(\bar{G})$, where constant $c>0$ is independent on u, spaces $B$ and $H$ are defined with the help of (5.3) and (5.4), operator $L=\left(\mathcal{L}, l_{0}, l_{1}\right)$ is defined with the help of operators $\mathcal{L}, l_{0}$, and $l_{1}$ of equation (5.1) and Cauchy condition (5.2)

Proof Each section $\Omega^{t}(0<t<1)$ divides domain $G$ info two subdomains $G^{t}$ and $\widetilde{G}^{t}$. Let $G^{t}$ be the subdomain for which the external normal $\boldsymbol{\nu}(x)$ to hypersurface $G^{t}$ at points $x \in \Omega^{t}$, as a part of the boundary $\partial G^{t}$, makes a sharp angle with the vector $\boldsymbol{r}(x)$, i. e. $r_{\nu}(x)>0$.

Boundary $\partial G^{t}$ of a set $G^{t}$ consists of a bottom base $\Omega^{0}$, top base $\Omega^{t}$ and a lateral surface $\Gamma^{t}=\Gamma \cap \overline{G^{t}}$, where $\overline{G^{t}}$ is a closure of $G^{t}$.

Let us integrate the expression $2 \mathcal{L}(x, \mathbf{D}) u \frac{\partial u}{\partial \boldsymbol{r}}$ over $G^{t}$, where 


$$
\frac{\partial u}{\partial \boldsymbol{r}}=u \boldsymbol{r}=\sum_{i=1}^{n} r_{i}(x) \frac{\partial u}{\partial x_{i}}
$$

In order to apply the Ostrogradsky formula, the principal part $2 \mathcal{L}_{0} u \frac{\partial u}{\partial \boldsymbol{r}}$ is represented in the form of divergence in the following way:

$$
\begin{aligned}
2(\mathcal{L}(x, \mathbf{D}) u, & \left.\frac{\partial u}{\partial \boldsymbol{r}}\right)_{L_{2}\left(G^{t}\right)}=\sum_{i, j, k=1}^{n} \int_{G^{t}}\left\{\left(a_{i j} r_{k} u_{x_{j}} u_{x_{k}}\right)_{x_{i}}\right. \\
& \left.-\left(a_{i j} r_{k} u_{x_{i}} u_{x_{j}}\right)_{x_{k}}+\left(a_{i j} r_{k} u_{x_{i}} u_{x_{k}}\right)_{x_{j}}\right\} \mathrm{d} x+\int_{G^{t}} \Phi(u) \mathrm{d} x,
\end{aligned}
$$

where $\Phi(u)$ is a bilinear form of the function $u$ and its first order derivatives:

$$
\begin{aligned}
\Phi(u)= & 2 \sum_{i, k=1}^{n} a_{i} r_{k} u_{x_{i}} u_{x_{k}}+2 \sum_{k=1}^{n} a_{0} r_{k} u u_{x_{k}} \\
& -\sum_{i, j, k=1}^{n}\left\{\left(a_{i j} r_{k}\right)_{x_{i}} u_{x_{j}} u_{x_{k}}-\left(a_{i j} r_{k}\right)_{x_{k}} u_{x_{i}} u_{x_{j}}+\left(a_{i j} r_{k}\right)_{x_{j}} u_{x_{i}} u_{x_{k}}\right\} .
\end{aligned}
$$

By virtue of the Ostrogradsky formula

$$
\begin{aligned}
& 2(\mathcal{L}(x, \mathbf{D}) u, u \boldsymbol{r})_{L_{2}\left(G^{t}\right)}=\mathcal{F}\left(G^{t}\right)+\int_{G^{t}} \Phi(u) \mathrm{d} x, \\
& \mathcal{F}\left(G^{t}\right)=\sum_{i, j=1}^{n} \int_{G^{t}} a_{i j}\left(u_{x_{j}} u_{\boldsymbol{r}} \nu_{i}-u_{x_{i}} u_{x_{j}} r_{\nu}+u_{x_{i}} u \boldsymbol{r} \nu_{j}\right) \mathrm{d} s .
\end{aligned}
$$

In order to estimate $\mathcal{F}\left(Q^{t}\right)$ we use a local Cartesian system

$$
\boldsymbol{\nu}, \boldsymbol{\tau}, \boldsymbol{\mu}, \boldsymbol{\tau}^{(1)}, \ldots, \boldsymbol{\tau}^{(n-3)} .
$$

In the mentioned local coordinate system one axis will go along vector $\boldsymbol{\nu}$, the other one - along the perpendicular to it vector $\tau \in \pi_{0}(x)$, where $\pi_{0}(x)$ is twodimensional plane containing vectors $\boldsymbol{\nu}$ and $\boldsymbol{r}$. Choose the other coordinates in the following way. Three hyperplanes passing through point $x$, where one plane is perpendicular to the vector $\boldsymbol{\nu}$, the second is $\operatorname{grad} u(x)$, the third one is $\boldsymbol{\tau}$, they intersect along dimension plane no less than $(n-3)$. In this intersection we choose orthogonal coordinate vectors $\boldsymbol{\tau}^{(1)}, \ldots, \boldsymbol{\tau}^{(n-3)}$. The last vector $\boldsymbol{\mu}(x)$ of this complete system is defined as a perpendicular vector to $\boldsymbol{\nu}(x), \boldsymbol{\tau}(x), \boldsymbol{\tau}^{(1)}, \ldots, \boldsymbol{\tau}^{(n-3)}$. By virtue of such choice

$$
u_{\boldsymbol{\tau}^{(s)}}=0, \quad s=1, \ldots, n-3 .
$$

By replacing the derivatives in the integrand along the new directions, $\mathcal{F}\left(G^{t}\right)$ can be written in the following form 


$$
\begin{aligned}
\mathcal{F}\left(G^{t}\right) & =\sum_{i, j=1}^{n} \int_{G^{t}} a_{i j}\left[\left(u_{\boldsymbol{\nu}} \nu_{j}+u_{\boldsymbol{\tau}} \tau_{j}+u_{\boldsymbol{\mu}} \mu_{j}\right)\left(u_{\boldsymbol{\nu}} r_{\nu}+u_{\boldsymbol{\tau}} r_{\tau}+u_{\boldsymbol{\mu}} r_{\mu}\right) \nu_{i}\right. \\
& -\left(u_{\boldsymbol{\nu} \nu_{i}}+u_{\boldsymbol{\tau}} \tau_{i}+u \boldsymbol{\mu} \mu_{i}\right)\left(u_{\boldsymbol{\nu} \boldsymbol{\nu}_{j}}+u_{\boldsymbol{\tau}} \tau_{j}+u_{\boldsymbol{\mu}} \mu_{j}\right) r_{\nu} \\
& \left.+\left(u_{\boldsymbol{\nu}} \boldsymbol{\nu}_{i}+u_{\boldsymbol{\tau}} \tau_{i}+u_{\boldsymbol{\mu}} \mu_{i}\right)\left(u_{\boldsymbol{\nu}} r_{\nu}+u_{\boldsymbol{\tau}} r_{\tau}+u_{\boldsymbol{\mu}} r_{\mu}\right) \boldsymbol{\nu}_{j}\right] \mathrm{d} s \\
& =\int_{\partial G^{t}} r_{\nu} \mathcal{L}_{0}(\boldsymbol{\nu}) u_{\boldsymbol{\nu}}^{2}+\left(2 r_{\tau} \mathcal{L}_{0}(\boldsymbol{\nu}, \boldsymbol{\tau})-r_{\nu} \mathcal{L}_{0}(\boldsymbol{\tau})\right) u_{\boldsymbol{\tau}}^{2} \\
& +\left(2 r_{\mu} \mathcal{L}_{0}(\boldsymbol{\nu}, \boldsymbol{\mu})-r_{\nu} \mathcal{L}_{0}(\boldsymbol{\mu})\right) u_{\boldsymbol{\mu}}^{2}+2 r_{\tau} \mathcal{L}_{0}(\boldsymbol{\nu}) u_{\boldsymbol{\nu}} u_{\boldsymbol{\tau}}+2 r_{\mu} \mathcal{L}_{0}(\boldsymbol{\nu}) u_{\boldsymbol{\nu}} u_{\boldsymbol{\mu}} \\
& \left.+2\left(r_{\mu} \mathcal{L}_{0}(\boldsymbol{\nu}, \boldsymbol{\tau})+r_{\tau} \mathcal{L}_{0}(\boldsymbol{\nu}, \boldsymbol{\mu})-r_{\nu} \mathcal{L}_{0}(\boldsymbol{\tau}, \boldsymbol{\mu})\right) u_{\boldsymbol{\tau}} u_{\boldsymbol{\mu}}\right] \mathrm{d} s \\
= & \int_{\partial G^{t}} \Phi^{0}(u) \mathrm{d} s
\end{aligned}
$$

where $\boldsymbol{\tau}=\left(\tau_{1}, \ldots, \tau_{n}\right), \boldsymbol{\mu}=\left(\mu_{1}, \ldots, \mu_{n}\right), r_{\zeta}=(\boldsymbol{r}, \boldsymbol{\zeta})=\sum_{k=1}^{n} r_{k} \zeta_{k}, \boldsymbol{\zeta} \in$ $\{\boldsymbol{\nu}, \boldsymbol{\tau}, \boldsymbol{\mu}\}$ in the coordinate system $x_{1}, \ldots, x_{n} ; \boldsymbol{r}=\left\{r_{\nu}, r_{\tau}, r_{\mu}, 0, \ldots, 0\right\}$ in the coordinate system $\boldsymbol{\nu}, \boldsymbol{\tau}, \boldsymbol{\mu}, \boldsymbol{\tau}^{(1)}, \ldots, \boldsymbol{\tau}^{(n-3)}$;

$$
\mathcal{L}_{0}(\boldsymbol{\zeta}, \boldsymbol{\xi})=\sum_{i, j=1}^{n} a_{i j}(x) \zeta_{i} \xi_{j}, \mathcal{L}_{0}(\boldsymbol{\zeta}, \boldsymbol{\zeta})=\mathcal{L}_{0}(\boldsymbol{\zeta})=\mathcal{L}_{0}(x, \boldsymbol{\zeta})
$$

By taking into account conditions (5.2) we rewrite integral (6.3) as the following sum

$$
\mathcal{F}\left(G^{t}\right)=\int_{\Omega^{t}} \Phi^{0}(u) \mathrm{d} s+\int_{\Omega^{0}} \Phi^{0}(u) \mathrm{d} s+\int_{\Gamma^{t}} \Phi^{0}(u) \mathrm{d} s=\mathcal{F}\left(\Omega^{t}\right)+\mathcal{F}\left(\Omega^{0}\right)+\mathcal{F}\left(\Gamma^{t}\right) .
$$

Values $\mathcal{F}\left(\Omega^{t}\right)$ and $\mathcal{F}\left(\Gamma^{t}\right)$ resemble each other by the absence of boundary conditions on $\Omega^{t}$ è $\Gamma^{t}$. In these integrals we consider the integrand as a quadratic form with respect to the derivatives $u_{\boldsymbol{\nu}}, u_{\boldsymbol{\tau}}, u_{\boldsymbol{\mu}}$. For estimation from below (4.1) we use the Sylvester criterion [5] with respect to quadratic form $\Phi^{0}(u)$, the matrix of which can be written in the form

$$
\left(\begin{array}{ccc}
r_{\nu} \mathcal{L}_{0}(\boldsymbol{\nu}) & r_{\tau} \mathcal{L}_{0}(\boldsymbol{\nu}) & r_{\mu} \mathcal{L}_{0}(\boldsymbol{\nu}) \\
r_{\tau} \mathcal{L}_{0}(\boldsymbol{\nu}) & 2 r_{\tau} \mathcal{L}_{0}(\boldsymbol{\nu}, \boldsymbol{\tau})-r_{\nu} \mathcal{L}_{0}(\boldsymbol{\tau}) & r_{\mu} \mathcal{L}_{0}(\boldsymbol{\nu}, \boldsymbol{\tau})+r_{\tau} \mathcal{L}_{0}(\boldsymbol{\nu}, \boldsymbol{\mu}) \\
& & -r_{\nu} \mathcal{L}_{0}(\boldsymbol{\tau}, \boldsymbol{\mu}) \\
r_{\mu} \mathcal{L}_{0}(\boldsymbol{\nu}) & r_{\mu} \mathcal{L}_{0}(\boldsymbol{\nu}, \boldsymbol{\tau})+r_{\tau} \mathcal{L}_{0}(\boldsymbol{\mu}) & 2 r_{\tau} \mathcal{L}_{0}(\boldsymbol{\nu}, \boldsymbol{\tau})-r_{\nu} \mathcal{L}_{0}(\boldsymbol{\mu}) \\
& -r_{\nu} \mathcal{L}_{0}(\boldsymbol{\tau}, \boldsymbol{\mu}) &
\end{array}\right) .
$$

Since for $x \in \Gamma^{t} \mathcal{L}_{0}(\boldsymbol{\nu})=0$, then for these $x$

$$
2 r_{\tau} \mathcal{L}_{0}(\boldsymbol{\nu}, \boldsymbol{\tau})-r_{\nu} \mathcal{L}_{0}(\boldsymbol{\tau})=-\frac{1}{r_{\nu}} \mathcal{L}_{0}(\boldsymbol{q})
$$

where vector $\boldsymbol{q}(x)=r_{\tau} \boldsymbol{\nu}(x)-r_{\nu} \boldsymbol{\tau}(x)$ represents $90^{\diamond}$ turn of the vector $\boldsymbol{r}(x)$ in the plane $\pi_{0}(x)$. Similarly, 


$$
\begin{aligned}
& 2 r_{\mu} \mathcal{L}_{0}(\boldsymbol{\nu}, \boldsymbol{\mu})-r_{\nu} \mathcal{L}_{0}(\boldsymbol{\mu})=-\frac{1}{r_{\nu}} \mathcal{L}_{0}(\boldsymbol{\chi}), \\
& r_{\mu} \mathcal{L}_{0}(\boldsymbol{\nu}, \boldsymbol{\tau})+r_{\tau} \mathcal{L}_{0}(\boldsymbol{\nu}, \boldsymbol{\mu})-r_{\nu} \mathcal{L}_{0}(\boldsymbol{\tau}, \boldsymbol{\mu})=-\frac{1}{r_{\nu}} \mathcal{L}_{0}(\boldsymbol{q}, \boldsymbol{\chi}),
\end{aligned}
$$

where vector $\boldsymbol{\chi}(x)=r_{\mu} \boldsymbol{\nu}(x)-r_{\nu} \boldsymbol{\mu}(x)$ represents $90^{\diamond}$ turn of the vector $\boldsymbol{r}(x)$ in the two-dimensional plane containing vectors $\boldsymbol{r}(x)$ and $\boldsymbol{\mu}(x)$. Thus, for any $x \in \Gamma^{t}$

$$
\Phi^{0}(u)=-\frac{1}{r_{\nu}}\left[\mathcal{L}_{0}(\boldsymbol{q}) u_{\boldsymbol{\tau}}^{2}+2 \mathcal{L}_{0}(\boldsymbol{q}, \boldsymbol{\chi}) u_{\boldsymbol{\tau}} u \boldsymbol{\mu}+\mathcal{L}_{0}(\boldsymbol{\chi}) u_{\boldsymbol{\mu}}^{2}\right](x) .
$$

According to presumption 1 ,

$$
\mathcal{L}_{0}(x ; \boldsymbol{q}(x)), \mathcal{L}_{0}(x ; \boldsymbol{\chi}(x)) \leqslant-c_{1}<0 .
$$

By applying the Cauchy-Bunyakovsky inequality [3] one can prove the inequality

$$
\mathcal{L}_{0}(x ; \boldsymbol{q}(x)) \mathcal{L}_{0}(x ; \boldsymbol{\chi}(x))-\mathcal{L}_{0}^{2}(x ; \boldsymbol{q}(x), \boldsymbol{\chi}(x)) \geqslant c_{2}>0 .
$$

Since $r_{\nu}>0, \mathcal{L}_{0}(x ; \boldsymbol{\nu}(x))=0$ and by virtue of the inequalities (6.5) and (6.6), we have that for all $x \in \Gamma^{t} \Phi^{0}(x) \geqslant 0$, i.e.

$$
\mathcal{F}\left(\Gamma^{t}\right)=\int_{\Gamma^{t}} \Phi^{0}(u) \mathrm{d} s \geqslant 0 .
$$

Let us make estimates for $\mathcal{F}\left(\Omega^{t}\right)$. We consider principal minors of the matrix of form $\Phi^{0}(u)$ in the case $x \in \Omega^{t}$ and rewrite them in an appropriate for the investigation form

$$
\begin{aligned}
d_{1}(x) & =r_{\nu}(x) \mathcal{L}_{0}(x ; \boldsymbol{\nu}(x)), \\
d_{2}(x) & =-\mathcal{L}_{0}(x ; \boldsymbol{\nu}(x)) \mathcal{L}_{0}(x ; \boldsymbol{q}(x)), \\
d_{3}(x) & =\frac{\mathcal{L}_{0}(\boldsymbol{\nu})}{r_{\nu}}\left|\begin{array}{ccc}
1 & r_{\nu} \mathcal{L}_{0}(\boldsymbol{\nu}) & r_{\mu} \mathcal{L}_{0}(\boldsymbol{\nu}) \\
0 & -\mathcal{L}_{0}(\boldsymbol{g}) & -\mathcal{L}_{0}(\boldsymbol{g}, \boldsymbol{\chi}) \\
0-\mathcal{L}_{0}(\boldsymbol{g}, \boldsymbol{\chi} & \mathcal{L}_{0}(\boldsymbol{\chi})
\end{array}\right|(x) \\
& =\frac{\mathcal{L}_{0}(x ; \boldsymbol{\nu}(x))}{r_{\nu}}\left[\mathcal{L}_{0}(x ; \boldsymbol{g}(x)) \mathcal{L}_{0}(x ; \boldsymbol{\chi}(x))-\mathcal{L}_{0}^{2}(\boldsymbol{g}(x), \boldsymbol{\chi}(x))\right] .
\end{aligned}
$$

From formulae (6.8)-(6.10) it is clear, that using the property of the vectors $\boldsymbol{g}(x)$ and $\chi(x)$ in the form of inequalities (6.5), (6.6) and the inequality

$$
r_{\nu}(x) \mathcal{L}_{0}(x ; \boldsymbol{\nu}(x)) \geqslant c_{5}>0,
$$

we have that for any $x \in \Omega^{t}$

$$
d_{p}(x) \geqslant c_{6}>0, p=1,2,3 .
$$

Thus, from inequalities (6.11) it follows that: 


$$
\mathcal{F}\left(\Omega^{t}\right)=\int_{\Omega^{t}} \Phi^{0}(u) \mathrm{d} s \geqslant c_{7} \int_{\Omega^{t}}\left[u_{\boldsymbol{\nu}}^{2}+u_{\boldsymbol{\tau}}^{2}+u_{\boldsymbol{\mu}}^{2}\right](x) \mathrm{d} s,
$$

where positive constant $c_{7}$ is independent of $u$. But

$$
u_{\boldsymbol{\nu}}^{2}+u_{\boldsymbol{\tau}}^{2}+u_{\boldsymbol{\mu}}^{2} \geqslant c_{8} \sum_{i=1}^{n} u_{x_{i}}^{2}
$$

or

$$
\mathcal{F}\left(\Omega^{t}\right) \geqslant c_{9} \sum_{i=1}^{n}\left\|u_{x_{i}}\right\|_{L_{2}\left(\Omega^{t}\right)}^{2} .
$$

Estimating expression $\mathcal{F}\left(\Omega^{0}\right)$ from above we obtain the inequality

$$
\mathcal{F}\left(\Omega^{0}\right)=\int_{\Omega^{0}} \Phi^{0}(u) \mathrm{d} s \leqslant c_{10}\left(\left\|l_{0} u\right\|_{H^{1}\left(\Omega^{0}\right)}^{2}+\left\|l_{1} u\right\|_{L_{2}\left(\Omega^{0}\right)}^{2}\right) .
$$

Using the Cauchy-Bunyakovsky inequality, it is easy to make estimates

$$
\begin{aligned}
& \left|\int_{\Omega^{t}} \Phi(u) \mathrm{d} s\right| \leqslant c_{11}\|u\|_{H^{1}\left(G^{t}\right)}^{2}, \\
& 2\left|(\mathcal{L} u, u \boldsymbol{r})_{L_{2}\left(G^{t}\right)}\right| \leqslant c_{12}\left(\|\mathcal{L} u\|_{L_{2}(G)}^{2}+\|u\|_{H^{1}\left(G^{t}\right)}^{2}\right) .
\end{aligned}
$$

Equalities (6.2)-(6.4) and estimates $(6.5)-(6.7),(6.12)-(6.14)$ together prove the inequality

$$
\sum_{i=1}^{n}\left\|u_{x_{i}}\right\|_{L_{2}\left(\Omega^{t}\right)}^{2} \leqslant c_{13}\|L u\|_{H}^{2}+\|u\|_{H^{1}\left(G^{t}\right)}^{2}
$$

Let us introduce the function $u$ into the left part of (6.15). For this purpose we integrate over $G^{t}$ the expression $\left(u^{2}\right) \boldsymbol{r}=2 u u \boldsymbol{r}$ and make appropriate transformations and estimates. As a result we obtain

$$
\int_{\Omega^{t}} u^{2} \mathrm{~d} s \leq c_{15}\left(\left\|l_{0} u\right\|_{H^{1}\left(\Omega^{0}\right)}^{2}+\left\|l_{1} u\right\|_{L_{2}\left(S_{4}\right)}^{2}+\|u\|_{H^{1}\left(G^{t}\right)}^{2}\right) .
$$

Adding inequalities (6.15) and (6.16) we obtain a new inequality to which it is possible to apply Gronuol inequality [7]. As a result we obtain the relation

$$
\int_{\Omega^{t}}\left(u^{2}+\sum_{i=1}^{n} u_{x_{i}}^{2}\right) \mathrm{d} s \leqslant c_{16}\|L u\|_{H}^{2},
$$

from which the needed energy inequality follows, if we apply the upper bound in the left part of (6.17). 
Cauchy Problem for a Linear Hyperbolic Eq. of the Second Order 287

\section{Proof of Theorem 3}

Now we will prove that operator $L$ is closable.

Lemma 1. Operator $L: B \rightarrow H$ of problem (5.1)-(5.2) allows closure $\bar{L}$.

Proof Let $u_{k} \in \mathcal{D}(L)$ and $u_{k} \underset{k \rightarrow \infty}{\stackrel{B}{\longrightarrow}} 0$. Since

$$
\left\|l_{0} u\right\|_{H^{1}\left(\Omega^{0}\right)}^{2} \leqslant \tilde{c}\|u\|_{B}, \quad\left\|l_{1} u\right\|_{L_{2}\left(\Omega^{0}\right)}^{2} \leqslant \tilde{c}\|u\|_{B}, \quad \tilde{c}>0,
$$

then it follows that $l_{0} u_{k} \rightarrow 0$ in $H^{1}\left(\Omega^{0}\right)$ and $l_{1} u_{k} \rightarrow 0$ in $L_{2}\left(\Omega^{0}\right)$ as $k \rightarrow \infty$. Let us consider a scalar product $\left(\mathcal{L} u_{k}, v\right)_{L_{2}(G)}$ for any function $v \in C_{0}^{\infty}(G)$, where $C_{0}^{\infty}(G)$ is a set of infinitely differentiable functions in $G$ with a compact support. If we transform it, we shall receive

$$
\left(\mathcal{L} u_{k}, v\right)_{L_{2}(G)}=\left(u_{k}, \sum_{i, j=1}^{n}\left(a_{i j} v_{x_{j}}\right)_{x_{i}}\right)_{L_{2}(G)}+\left(\sum_{i=1}^{n} a_{i}\left(u_{k}\right)_{x_{i}}+a_{0} u_{k}, v\right)_{L_{2}(G)} .
$$

Since

$$
u_{k} \underset{k \rightarrow \infty}{\stackrel{B}{\longrightarrow}} 0, \quad u_{k} \underset{k \rightarrow \infty}{\stackrel{L_{2}(G)}{\longrightarrow}} 0, \quad \sum_{i=1}^{n} a_{i} \frac{\partial}{\partial x_{i}} u_{k}+a_{0} u_{k} \underset{k \rightarrow \infty}{\stackrel{L_{2}(G)}{\longrightarrow}} 0,
$$

hence $\mathcal{L}(x, \mathbf{D}) u_{k} \underset{k \rightarrow \infty}{\stackrel{L_{2}(G)}{\longrightarrow}} 0$.

Theorem 5. If the presumption holds then for any $F \in H$ there exists the unique strong solution of the problem (5.1)-(5.2) and the estimate

$$
\|u\|_{B} \leqslant c\|F\|_{H} .
$$

is true.

Proof Estimate (7.1) follows from energy inequality (6.1) proved for operator $L$ of the problem (5.1)-(5.2). Uniqueness of a strong solution also follows from the energy inequality.

Now we prove the existence of a strong solution. For this purpose, according to corollary 1 it is necessary to show that $\overline{\mathcal{R}(L)}=H$. Consider a preliminary case, when operator $L$ has the form $L_{0}=\left(\mathcal{L}_{0}, l_{0}, l_{1}\right), \mathcal{D}\left(L_{0}\right)=\mathcal{D}(L)$ is a domain of its definition and $\mathcal{R}\left(L_{0}\right)$ is its range.

Let element $V(x)=\left(v(x), v_{0}(x), v_{1}(x)\right) \in H$ is orthogonal to $\mathcal{R}\left(L_{0}\right)$. We will show that $V(x)=0$. It means that the orthogonal addition to $\mathcal{R}\left(L_{0}\right)$ consists only of zero element, hence $\overline{\mathcal{R}\left(L_{0}\right)}=H$ [8]. Consider scalar product

$$
\left(L_{0} u, V\right)_{L_{2}(G)}=\left(\mathcal{L}_{0} u, v\right)_{L_{2}(G)}+\left(l_{0} u, v_{0}\right)_{H^{1}\left(\Omega^{0}\right)}+\left(l_{1} u, v_{1}\right)_{L_{2}\left(\Omega^{0}\right)},
$$

where $u \in \mathcal{D}\left(L_{0}\right)$. Let, in particular,

$$
u \in \mathcal{D}_{0}\left(L_{0}\right)=\left\{u \in \mathcal{D}\left(L_{0}\right) \mid l_{0} u=l_{1} u=0\right\} \subset \mathcal{D}(L) .
$$


Then the condition of the orthogonality becomes

$$
\left(\mathcal{L}_{0}(x, \mathbf{D}) u, v\right)_{L_{2}(G)}=0
$$

for all $u \in \mathcal{D}_{0}\left(L_{0}\right)$. In order to prove that $v=0$ in (7.3) we consider instead of $u$ a mollifier with variable step $J_{k} u$ given in the form

$$
J_{k} u(x)=\sum_{m=0}^{\infty} \psi_{m}(x) J_{\delta_{m k}} u(x),
$$

where $\psi_{m}(x)$ form the partition of the unity,

$$
J_{\delta_{m k}} u(x)=\frac{1}{\delta_{m k}^{n}} \int_{Q} \omega\left(\frac{x-y}{\delta_{m k}}\right) u(y) d y
$$

are the Sobolev averaging operators, $\delta_{m k}<2^{-4-m}[1]$.

Let $u \in C_{0}^{\infty}(G) \subset \mathcal{D}_{0}\left(L_{0}\right)$, then $J_{k} u \in C_{0}^{\infty}(G)$. Writing explicitly the left part of (7.3) and going to conjugate expressions we obtain

$$
\begin{aligned}
\left(\mathcal{L}_{0} J_{k} u, v\right)_{L_{2}(G)} & =\left(J_{k} \mathcal{L}_{0} u, v\right)_{L_{2}(G)}+\left(\mathcal{L}_{0} J_{k} u-J_{k} \mathcal{L}_{0} u, v\right)_{L_{2}(G)} \\
& =\left(\mathcal{L}_{0} u, J_{k}^{*} v\right)_{L_{2}(G)}+(R u, v)_{L_{2}(G)} \\
& =\left(u, \mathcal{L}_{0} J_{k}^{*} v\right)_{L_{2}(G)}+\left(u, R_{0}^{*} v\right)_{L_{2}(Q)}-\sum_{i=1}^{n}\left(u, \frac{\partial}{\partial x_{i}} R_{i}^{*} v\right)_{L_{2}(G)},
\end{aligned}
$$

where $J_{k}^{*}$ is adjoint to operator $J_{k}$ and it can be presented as

$$
J_{k}^{*} u(x)=\sum_{m=0}^{\infty} J_{\delta_{m k}}\left(\psi_{m} u\right)(x),
$$

$R=\mathcal{L}_{0} J_{k}-J_{k} \mathcal{L}_{0}$ is commutator which can be given in the form

$$
\begin{aligned}
& R u=R_{0} u+\sum_{i=1}^{n} R_{i} u_{x_{i}} \\
& R_{0} u=\sum_{m=0}^{\infty} \sum_{i, j=1}^{n}\left\{\frac{\partial a_{i j}}{\partial x_{i}} \frac{\partial \psi_{m}}{\partial x_{j}} J_{\delta_{m k}} u+a_{i j} \frac{\partial^{2} \psi_{m}}{\partial x_{i} \partial x_{j}} J_{\delta_{m k}} u\right. \\
& \left.-\psi_{m} \frac{1}{\delta_{m k}^{n}} \int_{G} \frac{\partial}{\partial y_{j}}\left[\omega\left(\frac{x-y}{\delta_{m k}}\right)\left(\frac{\partial a_{i j}(x)}{\partial x_{i}}-\frac{\partial a_{i j}(y)}{\partial y_{i}}\right)\right] u(y) d y\right\} . \\
& R_{i} \frac{\partial u}{\partial x_{i}}=\sum_{m=0}^{\infty} \sum_{j=1}^{n}\left\{2 a_{i j} \frac{\partial \psi_{m}}{\partial x_{j}} J_{\delta_{m k}} \frac{\partial u}{\partial x_{i}}\right. \\
& \left.\quad-\psi_{m} \frac{1}{\delta_{m k}^{n}} \int_{Q} \frac{\partial}{\partial y_{j}}\left[\omega\left(\frac{x-y}{\delta_{m k}}\right)\left(a_{i j}(x)-a_{i j}(y)\right)\right] \frac{\partial u}{\partial y_{i}} \mathrm{~d} y\right\} .
\end{aligned}
$$


Taking in account (7.4), we can write equality (7.3) for any function $u \in$ $C_{0}^{\infty}(G)$ in the following form

$$
\left(u, \mathcal{L}_{0} J_{k}^{*} v+R_{0}^{*} v-\sum_{i=1}^{n} \frac{\partial}{\partial x_{i}} R_{i}^{*} v\right)_{L_{2}(G)}=0 .
$$

Note, that (7.5) can be extended to functions $u \in L_{2}(G)$ by limit passage.

We return to equality (7.3) where $u$ is taken as $J_{k} u, u \in \mathcal{D}_{0}\left(L_{0}\right)$. Using the form of integrated transfer operator we transfer operator of differentiation from $u$ to $v$ and obtain

$$
\left(u, \mathcal{L}_{0} J_{k}^{*} v+R_{0}^{*} v-\sum_{i=1}^{n} \frac{\partial}{\partial x_{i}} R_{i}^{*} v\right)_{L_{2}(G)}+M(u, v ; \partial G(y))=0,
$$

where we denote by $M(u, v ; \partial G(y))$ boundary terms which result from integrating by parts in the expression $\left(\mathcal{L}_{0} u, J_{k}^{*} v\right)_{L_{2}(G)}+(R u, v)_{L_{2}(G)}$. By comparing (7.5) and (7.6), we see that the following equality is fulfilled

$$
M(u, v ; \partial G(y))=0 .
$$

By varying the function $u$ within the limit of the set $\mathcal{D}_{0}(L)$ one can show that (7.7) is fulfilled for any $u \in \mathcal{D}_{0}\left(L_{0}\right)$ if and only if $v \in L_{2}(G)$ is such that

$$
\left.J_{k}^{*} v\right|_{\Gamma}=0,\left.\quad R_{i}^{*} v\right|_{\Gamma}=0, \quad i=0, \ldots, n .
$$

Let us denote by $\tilde{G}^{t}$ cofactor to $G^{t} \cup \Omega^{t}$ of the domain $G$. Here the value $t$ is chosen so that $\tilde{G}^{t}$ is a convex set with respect to the vector field $\Re$ throughout the set $G$. Let us introduce $\left(\partial \tilde{G}^{t}\right)^{-}=\left\{x \in \partial \tilde{G}^{t} \mid r_{\nu}(x)<0\right\}$, where $\boldsymbol{\nu}(x)$ is a unit vector of the external normal. Similarly, $\left(\partial \tilde{G}^{t}\right)^{+}=\left\{x \in \partial \tilde{G}^{t} \mid r_{\nu}(x)>0\right\}$. By $I v(x)$ we denote a line integral

$$
I v(x)=\int_{\tilde{x}}^{x} J_{k}^{*} v \mathrm{~d} s,
$$

where integration is fulfilled along the curve $\rho$, to which the vector field $\Re$ is tangent, $x$ and $\tilde{x}$ are on this curve and $\tilde{x} \in\left(\partial \tilde{G}^{t}\right)^{-}, x \in \overline{\tilde{G}^{t}}$. From definition of the integral $I$ and conditions (7.8) it follows that

$$
\left.J_{k}^{*} v\right|_{\left(\partial \tilde{G}^{t}\right)^{+}}=0,\left.I v\right|_{\left(\partial \tilde{G}^{t}\right)^{-}}=0, \quad J_{k}^{*} v(x)=\frac{\partial}{\partial \boldsymbol{r}} I v(x) .
$$

If in (7.5) we choose $u$ as

$$
u(x)= \begin{cases}\operatorname{Iv}(x), & x \in \tilde{G}^{t}, \\ 0, & x \in G^{t}\end{cases}
$$

then as a result we obtain 


$$
\sum_{i, j=1}^{n} \int_{\tilde{G}^{t}} I v \frac{\partial}{\partial x_{j}}\left(a_{i j} \frac{\partial^{2}}{\partial x_{i} \partial \boldsymbol{r}} I v\right) d x=\sum_{i=1}^{n}\left(I v, \frac{\partial}{\partial x_{i}} R_{i}^{*} v\right)_{L_{2}\left(\tilde{G}^{t}\right)}-\left(I v, R_{0}^{*} v\right)_{L_{2}\left(\tilde{G}^{t}\right)} .
$$

Let us integrate by parts in the left part of (7.10) using the Ostrogradsky formula. For this purpose we transform the subintegral expression into the following divergent form

$$
\sum_{i, j=1}^{n} I v \frac{\partial}{\partial x_{i}}\left(a_{i j} \frac{\partial^{2}}{\partial x_{j} \partial \boldsymbol{r}} I v\right)=\sum_{i, j=1}^{n} \frac{\partial}{\partial x_{i}}\left(a_{i j} I v \frac{\partial^{2}}{\partial x_{j} \partial \boldsymbol{r}} I v\right)-\frac{1}{2} \frac{\partial}{\partial \boldsymbol{r}} \Psi_{0}(v)-\Psi_{1}(v),
$$

where we denote

$$
\begin{aligned}
& \Psi_{0}(v)=\sum_{i, j=1}^{n} a_{i j} \frac{\partial}{\partial x_{i}} I v \frac{\partial}{\partial x_{j}} I v \\
& \Psi_{1}(v)=-\frac{1}{2} \sum_{i, j=1}^{n} \frac{\partial a_{i j}}{\partial \boldsymbol{r}} I v \frac{\partial}{\partial x_{j}} I v+\sum_{i, j, k=1}^{n} a_{i j} \frac{\partial r_{k}}{\partial x_{j}} \frac{\partial}{\partial x_{i}} I v \frac{\partial}{\partial x_{k}} I v .
\end{aligned}
$$

By virtue of conditions (7.9)

$$
\int_{\tilde{G}^{t}} \sum_{i, j=1}^{n} \frac{\partial}{\partial x_{i}}\left(a_{i j} I v \frac{\partial^{2}}{\partial x_{j} \partial \boldsymbol{r}} I v\right) \mathrm{d} x=0 .
$$

Taking into account (7.11) and (7.12), the equation (7.10) can be written as follows:

$-\int_{\partial \tilde{G}^{t}} \Psi_{0}(v) r_{\nu} \mathrm{d} s=2 \int_{\tilde{G}^{t}} \Psi_{1}(v) \mathrm{d} x-2\left(I v, R_{0}^{*} v\right)_{L_{2}\left(\tilde{G}^{t}\right)}+2 \sum_{i=1}^{n}\left(\frac{\partial}{\partial x_{i}} I v, R_{i}^{*} v\right)_{L_{2}\left(\tilde{G}^{t}\right)}$.

In the point $x \in \Omega^{t}$ choose the local Cartesian coordinate system $\left\{\boldsymbol{\nu}, \boldsymbol{\tau}^{2}\right.$, $\left.\ldots, \tau^{n}\right\}$. In this system the derivative representation is true

$$
\frac{\partial}{\partial x_{i}} I v=\frac{\partial}{\partial \boldsymbol{\nu}} I v \nu_{i}+\frac{\partial}{\partial \tau^{2}} I v \tau_{i}^{2}+\ldots+\frac{\partial}{\partial \boldsymbol{\tau}^{n}} I v \tau_{i}^{n} .
$$

But

$$
\frac{\partial}{\partial \boldsymbol{r}} I v=\frac{\partial}{\partial \boldsymbol{\nu}} I v r_{\nu}+\frac{\partial}{\partial \boldsymbol{\tau}^{2}} I v r_{\tau^{2}}+\ldots+\frac{\partial}{\partial \boldsymbol{\tau}^{n}} I v r_{\tau^{n}}
$$

Hence,

$$
\frac{\partial}{\partial x_{i}} I v=\frac{\nu_{i}}{r_{\nu}} J_{k}^{*} v+\left(\tau_{i}^{2}-\frac{r_{\tau^{2}}}{r_{\nu}} \nu_{i}\right) \frac{\partial}{\partial \tau^{2}} I v+\ldots+\left(\tau_{i}^{n}-\frac{r_{\tau^{n}}}{r_{\nu}} \nu_{i}\right) \frac{\partial}{\partial \boldsymbol{\tau}^{n}} I v=\frac{\nu_{i}}{r_{\nu}} J_{k}^{*} v .
$$

Thus,

$$
-\int_{\Omega^{t}} \Psi_{0}(v) r_{\nu} \mathrm{d} s=-\int_{S^{t}} \frac{1}{r_{\nu}}\left(J_{k}^{*} v\right)^{2}{ }_{0}(x ; \boldsymbol{\nu}) \mathrm{d} s \geqslant c_{1}\left\|J_{k}^{*} v\right\|_{L_{2}\left(\Omega^{t}\right)}^{2} .
$$


Now we consider $\Psi_{0}(v)$ on $\left(\partial \tilde{G}^{t}\right)^{+}$. Here for almost all $x$ in the local Cartesian coordinate system $\left\{\boldsymbol{r}, \boldsymbol{q}^{2}, \ldots, \boldsymbol{q}^{n}\right\}$ the derivatives representation is true

$$
\frac{\partial}{\partial x_{i}} I v=\frac{\partial}{\partial \boldsymbol{r}} I_{v} r_{i}+\frac{\partial}{\partial \boldsymbol{q}^{2}} I v q_{i}^{2}+\ldots+\frac{\partial}{\partial \boldsymbol{q}^{n}} I v q_{i}^{n}=\sum_{k=2}^{n} q_{i}^{k} \frac{\partial}{\partial \boldsymbol{q}^{k}} I v .
$$

According to Proposition 2 and by virtue of representation (7.15) and the fact that $r_{\nu} \geqslant \delta>0$, for $x \in\left(\partial \tilde{G}^{t}\right)^{+},(\boldsymbol{r}, \boldsymbol{\theta})=0$,

$$
\boldsymbol{\theta}=\left(\sum_{k=2}^{n} q_{1}^{k} \frac{\partial}{\partial \boldsymbol{q}^{k}} I v, \ldots, \sum_{k=2}^{n} q_{n}^{k} \frac{\partial}{\partial \boldsymbol{q}^{k}} I v\right)
$$

we have

$$
\begin{aligned}
& -\int_{\left(\partial \tilde{G}^{t}\right)^{+}} \Psi_{0}(v) r_{\nu} \mathrm{d} s=-\sum_{k=2}^{n} \int_{\left(\partial \tilde{G}^{t}\right)^{+}} r_{\nu}(x) \mathcal{L}_{0}(x, \boldsymbol{\theta}) \mathrm{d} s \\
& \geqslant c_{2} \sum_{k=2}^{n} \int_{\left(\partial \tilde{G}^{t}\right)^{+}}\left(\sum_{i=1}^{n} q_{i}^{k} \frac{\partial}{\partial \boldsymbol{q}^{k}} I v\right)^{2} \mathrm{~d} s \geqslant c_{3} \sum_{i=1}^{n} \int_{\left(\partial \tilde{G}^{t}\right)^{+}}\left(\frac{\partial}{\partial x_{i}} I v\right)^{2} \mathrm{~d} s .
\end{aligned}
$$

Now we return back to equation (7.15). We estimate its left part from below using the Cauchy-Bunyakovsky inequality. Then in order to apply the Gronuol inequality, along with integral $I v$, we introduce the integral

$$
\hat{I} v(x)=\int_{x}^{\hat{x}} J_{k}^{*} v \mathrm{~d} s,
$$

where integration is fulfilled along the same lines as in $I v$. Here $\hat{x} \in\left(\partial \tilde{G}^{t}\right)^{+}$. It follows from the definitions of $I$ and $\hat{I}$ that they are connected by the relation

$$
I v(x)+\hat{I}(x)=\hat{I} v(\tilde{x}) .
$$

From (7.15) by virtue of (7.14) and (7.16) and after the substitution of $I$ for $\hat{I}$ we obtain the inequality

$$
\begin{aligned}
c_{1}\left\|J_{k}^{*} v\right\|_{L_{2}\left(S^{t}\right)}^{2} & +c_{3} \sum_{i=1}^{n} \int_{\left(\partial \tilde{G}^{t}\right)^{-}}\left(\frac{\partial}{\partial x_{i}} \hat{I} v\right)^{2}(\tilde{x}) \beta(\tilde{x}) \mathrm{d} s \\
\leqslant & \mid \sum_{i=1}^{n}\left(\frac{\partial}{\partial x_{i}} \hat{I} v(\tilde{x})-\frac{\partial}{\partial x_{i}} \hat{I} v(x), R_{i}^{*} v\right)_{L_{2}\left(\tilde{G}^{t}\right)} \\
& -\left(\hat{I} v(\tilde{x})-\hat{I} v(x), R_{0}^{*} v\right)_{L_{2}\left(\tilde{G}^{t}\right)}-\int_{\tilde{G}^{t}} \Psi_{1}(v) d x \mid .
\end{aligned}
$$


Function $\beta(\tilde{x})$ resulted from the substitution of the integration domain and it is easy to prove that $\beta(\tilde{x}) \geqslant \tilde{c}>0$.

Along with (7.17) we consider the inequality

$$
\frac{1}{2} \int_{\left(\partial \tilde{G}^{t}\right)^{-}}(\hat{I} v)^{2}(\tilde{x}) \nu_{r}(\hat{x}) \beta_{1}(\tilde{x}) \mathrm{d} s=\int_{\tilde{G}^{t}} J_{k}^{*} v(x)[\hat{I} v(\tilde{x})-\hat{I} v(x)] \mathrm{d} x,
$$

which results from the relation

$$
\frac{1}{2} \frac{\partial}{\partial \boldsymbol{r}}(I v)^{2}(x)=J_{k}^{*} v(x) \operatorname{Iv}(x)
$$

by integrating it over the subdomain $\tilde{G}^{t}$. Here also $\beta_{1}(\tilde{x}) \geqslant \tilde{c}>0$ for some constant $\tilde{c}$. From (7.18) the inequality

$$
c_{4}\|\hat{I} v\|_{L_{2}\left(\left(\partial \tilde{G}^{t}\right)^{-}\right)}^{2} \leqslant c_{5} \int_{\partial \tilde{G}^{t}} J_{k}^{*} v(x)[\hat{I} v(\tilde{x})-\hat{I} v(x)] d x .
$$

If we sum inequalities (7.17) and (7.19), we obtain a non-negative expression in the left part of the obtained inequality. In order to estimate the right part we apply the Cauchy-Bunyakovsky inequality. Here we also use the estimates [10]

$$
\left\|R_{i}^{*} v\right\|_{L_{2}\left(\tilde{G}^{t}\right)}^{2} \leqslant c_{5}\|v\|_{L_{2}\left(\tilde{G}^{t}\right)} \quad(i=0,1, \ldots, n) .
$$

As a result we obtain

$$
\begin{aligned}
&\left\|J_{k}^{*} v\right\|_{L_{2}\left(\Omega^{t}\right)}+\|\hat{I} v\|_{L_{2}\left(\left(\partial \tilde{G}^{t}\right)^{-}\right)}+\sum_{i=1}^{n}\left\|\frac{\partial}{\partial x_{i}} \hat{I} v\right\|_{L_{2}\left(\left(\partial \tilde{G}^{t}\right)^{-}\right)} \\
& \leq c_{6}\left(\varepsilon_{0}\right)\left[\int_{\tilde{G}^{t}}\left\{(\hat{I} v)^{2}+\sum_{i=1}^{n}\left(\frac{\partial}{\partial x_{i}} \hat{I} v\right)^{2}\right\}^{1 / 2}(\tilde{x}) \mathrm{d} x\right. \\
&\left.+\left\|J_{k}^{*} v\right\|_{L_{2}\left(\tilde{G}^{t}\right)}+\|\hat{I} v\|_{L_{2}\left(\tilde{G}^{t}\right)}+\sum_{i=1}^{n}\left\|\frac{\partial}{\partial x_{i}} \hat{I} v\right\|_{L_{2}\left(\tilde{G}^{t}\right)}\right]+\varepsilon_{0}\|v\|_{L_{2}\left(\tilde{G}^{t}\right)},
\end{aligned}
$$

where $c_{6}\left(\varepsilon_{0}\right)$ increases inversely proportional to $\varepsilon_{0}>0$. The first terms of (7.20) can be estimated as follows

$$
\begin{aligned}
\int_{\tilde{Q}^{t}}\left\{(\hat{I} v)^{2}+\sum_{i=1}^{n}\left(\frac{\partial}{\partial x_{i}} \hat{I} v\right)^{2}\right\}^{1 / 2}(\tilde{x}) \mathrm{d} x & \\
& \leqslant c_{7} \tau\left[\|\hat{I} v\|_{L_{2}\left(\left(\partial \tilde{G}^{t}\right)^{-}\right)}+\sum_{i=1}^{n}\left\|\frac{\partial}{\partial x_{i}} \hat{I} v\right\|_{L_{2}\left(\left(\partial \tilde{G}^{t}\right)^{-}\right)}\right]
\end{aligned}
$$

where $\tau$ decreases as the domain of $\tilde{G}^{t}$ on the lines $\rho$ decreases, i.e. $\Omega^{t}$ is chosen so that lines $\rho$ of the domain $\tilde{G}^{t}$ are short enough. Choose $\tau_{0}$ so that 


$$
2 c_{7} c_{6}\left(\varepsilon_{0}\right) \tau_{0} \leqslant 1, \quad 4 T^{1 / 2} \varepsilon_{0} e^{2 c_{6}\left(\varepsilon_{0}\right) \tau_{0}} \leqslant 1,
$$

where $T$ is maximum length of lines $\rho$ throughout the domain $\tilde{G}_{i_{0}}$. Now for all $0 \leqslant \tau \leqslant \tau_{0}$ the following inequality is valid

$$
w(t) \leqslant 2 c_{6}\left(\varepsilon_{0}\right) \int_{\tilde{x}}^{\hat{x}} w(x) \mathrm{d} s+2 \varepsilon_{0}\|v\|_{L_{2}\left(\tilde{G}^{t}\right)}
$$

by virtue of (7.21) and the fact that

$$
w(t) \leqslant\left\|J_{k}^{*} v\right\|_{L_{2}\left(\Omega^{t}\right)}+\|\hat{I} v\|_{L_{2}\left(\Omega^{t}\right)}+\sum_{i=1}^{n}\left\|\frac{\partial}{\partial x_{i}} \hat{I} v\right\|_{L_{2}\left(\left(\partial \tilde{G}^{t}\right)^{-}\right)},
$$

where

$$
w(t)=\left\|J_{k}^{*} v\right\|_{L_{2}\left(\Omega^{t}\right)}+\|\hat{I} v\|_{L_{2}\left(\Omega^{t}\right)}+\sum_{i=1}^{n}\left\|\frac{\partial}{\partial x_{i}} \hat{I} v\right\|_{L_{2}\left(\Omega^{t}\right)} .
$$

Applying the Gronuol inequality to inequality (7.22) we obtain

$$
w(t) \leqslant \varepsilon_{0} e^{2 c_{6}\left(\varepsilon_{0}\right) \tau_{0}}\|v\|_{L_{2}\left(\tilde{G}^{t}\right)}
$$

or for $\tilde{G}^{t_{0}} \subset \tilde{G}_{t_{0}}$

$$
\frac{1}{T^{1 / 2}}\left\|J_{k}^{*} v\right\|_{L_{2}\left(\tilde{G}^{t_{0}}\right)} \leqslant\left\|J_{k}^{*} v\right\|_{L_{2}\left(\Omega^{t}\right)} \leqslant \frac{1}{4 T^{1 / 2}}\|v\|_{L_{2}\left(\tilde{G}^{t_{0}}\right)} .
$$

If we pass to the limit when $k \rightarrow \infty$ in $(7.23)$, we have $\|v\|_{L_{2}\left(G^{\tau_{0}}\right)} \leqslant 0$, i.e. $v=0$ in $L_{2}\left(G^{t_{0}}\right)$. Continuing this process further, in finite number of steps we prove that $v=0$ in upper convex over $\Re$ set $\tilde{G}_{i_{0}}$. Moving further from the top downwards in finite number of steps we show that $v=0$ throughout the whole domain $G$.

Let us return back to (7.2). We have the relation

$$
\left(l_{0} u, v_{0}\right)_{H^{1}\left(\Omega^{0}\right)}+\left(l_{1} u, v_{1}\right)_{L_{2}\left(\Omega^{0}\right)}=0, \quad u \in \mathcal{D}(L) .
$$

Since $l_{0}$ and $l_{1}$ are linearly independent and sets $\left\{l_{0} u\right\},\left\{l_{1} u\right\}$ are dense in $H^{1}\left(\Omega^{0}\right)$ ) and $\left.L_{2}\left(\Omega^{0}\right)\right)$, respectively, if $u$ runs through the whole set $\mathcal{D}(L)$, then equality (7.24) implies that $v_{0}=0$ in $\left.H^{1}\left(\Omega^{0}\right)\right)$ and $v_{1}=0$ in $L_{2}\left(\Omega^{0}\right)$. Thus it is proved that density of $\mathcal{R}\left(L_{0}\right)$ is in $H$.

In the general case the fact that density of $\mathcal{R}(L)$ is in $H$ can be proved by continuing along the parameter [9].

\section{References}

[1] V.I. Burenkov. Sobolev spaces on domains. Stuttgart-Leipzig, 1998.

[2] A.A. Dezin. To the general theory of boundary problems. Mathematical Digest, $\mathbf{1 0 0}(2), 117-180,1980$. 
[3] R.E. Edwards. Functional analysis. Theory and application. Holt, Rinehard and Wiston, 1965.

[4] L. Gairding. Cauchy's problem for hyperbolic equations. Winter ana Spring Quarters. University of Chicago, 1957.

[5] Z. F. Gantmacher. Theory of Matrieces. Moscow, 1967. (in Russian)

[6] S. Gindikin and L. Volevich. Mixed problem for partial differential equations with quasihomogeneous principal part. Translation of Mathematical Monographs. Amer. Math. Soc. Providence. 147. R1

[7] Ph. Hartman. Ordinary Differential Equations. New York-London-Sydney, 1964.

[8] L.V. Kantorovich and G.P. Akilov. Functional analysis. Nauka, Moscow, 1965. (in Russian)

[9] V. I. Korzyuk. Mixed problem for some nonstationary equations with disconnected coefficient. Differential Equation, 6(2), 343-357, 1970.

[10] V. I. Korzyuk. Fridrichs lemma for mollifiers with variable step. Vestnik of Belorussian State University, 1(2), 32-36, 1996.

[11] V. I. Korzyuk. Method of energy inequations and mollifiers. Vestnik of Belorussian State University, 1(3), 55-67, 1996. 